\title{
APCO Project 25 Wireless Data Services for Smaller Public Safety Agencies
}

\author{
Ivan Elhart, W. Thomas Miller III, and Andrew L. Kun \\ University of New Hampshire \\ Dept. of Electrical \& Computer Engineering \\ Kingsbury Hall / 33 College Road \\ Durham, NH 03824, USA, \\ (603)862 1357, Fax: (603)862 1832 \\ ivan.elhart@unh.edu, tom.miller@unh.edu, andrew.kun@unh.edu
}

\begin{abstract}
Digital data messages are very important in modern communication systems and the advanced data technologies that rely on the Internet Protocol have opened the door to a wide range of IP-based applications and services. With this trend in mind, we have implemented a system that will allow smaller law enforcement agencies to enable data services in their cruisers in a cost effective way, with a high level of reliability. Towards this goal, we have implemented an inexpensive Software Defined APCO Project 25 Data Base Station that utilizes the standard IP network interface. This paper describes the overall design of the data base station, its implementation in the preexisting radio network infrastructure, and the testing process. Laboratory tests have produced promising and encouraging results prior to real world deployment.
\end{abstract}

\section{INTRODUCTION}

Today, modern communication systems utilize digital data messages to access, store, and manipulate desired information. Those systems allow for a wide range of data services along with traditional voice communication. A well known example of such a system is a cellular phone network that supports Short Message Service (SMS), Multimedia Messaging Service (MMS), and advanced data services. Cellular data technologies (e.g. General Packet Radio Service - GPRS) use Internet Protocol (IP) for the routing and exchange of data packets. This feature has opened the door to broad IP-based applications and services such as WWW, email, navigation, and even TV for mobile phones.

In addition, cellular networks combined with other wireless/wired networks have been used for various public services ranging from fleet management, navigation, and telemetry to tracking, guiding, and providing additional information to users. Most of these services would be beneficial for law enforcement agencies in organizing and tracking their personnel.

However, they are not widely used among public safety agencies because first responders cannot rely on the survivability and redundancy of public services. Even with a vision that commercial systems will augment or even completely replace public safety networks in the future, public safety and mission-critical operations should not count on commercial networks unless they satisfy high safety requirements of reliability, priority in traffic, and quality of service [1] [2].

Law enforcement communication primarily depends on Land Mobile Radio (LMR) and the spectrum reserved by the Federal Communication Commission for public safety use. LMR systems were initially designed to operate in analog mode and to provide only voice communication. Later, standards for digital communication allowed analog radio systems to be replaced with digital ones. Still, digital LMR systems use voice as the primary way to exchange information, and most of them use voice as the only way for communication. Relatively few currently deployed LMR voice systems have data capabilities sufficient to support IP over the air [1].

Data functionality was added to public safety LMR by implementing a set of common technical standards for digital communication, known as the Association of PublicSafety Communication Officials (APCO) Project 25 (P25). However, this data feature introduced a new piece of equipment to the system called a Data Base Station. The high complexity of commercially available P25 data capable base stations raises the cost of such equipment and its installation. This is the main reason why P25 data systems are not more widespread.

The New Hampshire Department of Safety (NHDS) was among the first agencies to implement a state wide P25 radio infrastructure that supports mixed voice and data communication. The NHDS benefits from the system by processing approximately 20,000 data queries per month over the same LMR channels used for voice transmissions. Systems like the one used by the NHDS may cost hundreds 
of thousands of dollars, which is not affordable by smaller agencies due to their limited budget. However, nearly all local police departments in $\mathrm{NH}$ are already equipped with P25-compliant mobile radios, but they lack the expensive base stations to support data traffic. Currently, local departments utilize only voice communication to provide their cruisers with vehicle record information, which is illustrated in Figure 1.

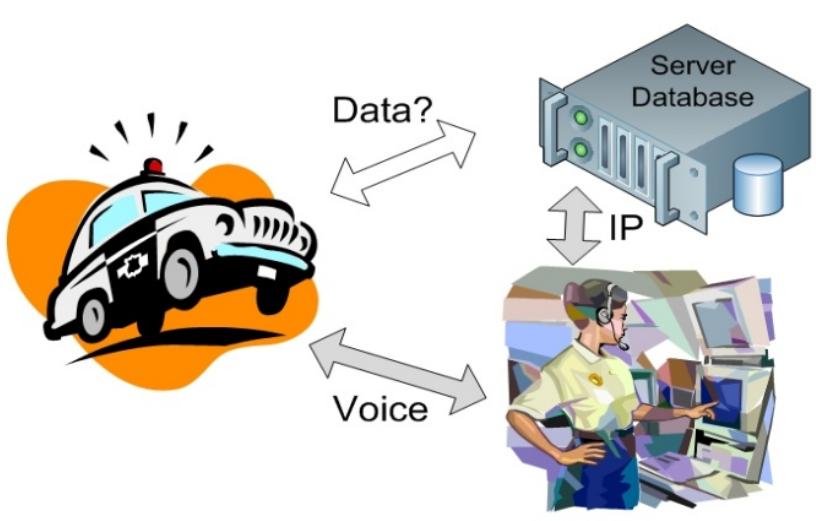

Figure 1 - Current state of communication in local NH police departments

The goal of this work was to develop a system that would rely on the existing P25 compliant networks and allow small local law enforcement agencies to enable data services in their cruisers. In order to achieve our goal, we have implemented an inexpensive Software Defined APCO Project 25 Data Base Station that implements a standard IP network interface.

\section{RELATED WORK}

APCO established Project 25, a set of common technical standards, to initially address the radio interoperability problem and make the usage of scarce radio frequencies more efficient. Communication between mobile, portable, and base radios is specified through an open digital protocol called the Common Air Interface (CAI) [3]. This interface adds data packets to the LMR and describes both voice and data transmissions over a radio channel.

Since P25 has been present for a while, many third party companies have worked on the implementation and development of P25 Base Stations. Those companies, such as Tyco Electronics [4] and Etherstack [5], provide a comprehensive range of software-defined P25 voice and data solutions. Tyco Electronics developed the VIDA communication network that supports a line of communication systems including $\mathrm{P} 25^{\mathrm{IP}}$. This system enhances the P25 standards with the advantages of the IPbased infrastructure. Etherstack offers several variants of APCO P25 compliant base stations. These variants are written in highly portable $\mathrm{ANSI} \mathrm{C} / \mathrm{C}++$ with a layered architecture abstracted from the underlying hardware platform and operating system. Because these solutions are software-based they are highly flexible, so the reconfiguration or updating of the system can be easily achieved. Still, these systems keep a high level of complexity by integrating voice and data signaling, by supporting transparent hand-off across multiple repeaters and trunked channels, and so forth. Such complexity is not necessary for small public agencies that already have fully operational voice networks and operate on a single conventional channel. They only need an inexpensive, stand alone data capable base station.

Work on a single, stand alone P25 data base station started at the University of New Hampshire with the overall design and development of the software based P25 data packet transmitter [6]. This work demonstrates how IP packets can be broadcast over the air using a desktop computer and an analog radio. The desktop computer runs digital signal processing software that receives IP packets through a standard Ethernet interface. When IP packets are received by the application, they are encoded into CAI data packets which are then passed to an analog radio as analog waveforms through the computer's sound card. The waveforms are broadcast to the air using the analog transmitter on a predefined channel. A mobile digital radio, which can be found in most $\mathrm{NH}$ public safety vehicles, tuned to the same frequency captures the waveforms and decodes sent IP packets. With this transmitter small departments can deliver additional information to the cruisers in a cost effective way. Also, the usage of a single digital radio for both voice and data traffic reduces the cost of providing data services to the vehicles without involving additional wireless devices.

\section{SOFTWARE-BASED APCO PROJECT 25 DATA RECEIVER AND BASE STATION LOGIC}

In order to support fully operational voice and data traffic for local police departments, we have implemented an APCO P25 data base station and incorporated it with a preexisting voice communication line. This is illustrated in Figure 2. Both voice and data packets are transmitted and received on the same digital radio channel using the Time Division Multiple Access (TDMA) technique and 4-level frequency modulation [7]. This approach, where the same radio channel is used for both voice and data signaling and where data messages are handled by separate data equipment, requires no additional communication devices and no modifications in the preexisting voice line.

The reception and transmission of data packets is performed using a desktop computer and an analog radio. On the reception side, the analog radio receives both voice and data CAI waveforms. These analog waveforms are then passed to a DSP application through the computer's sound card input. The application decodes digitally sampled versions of data packets into sent IP packets, while the voice signals are discarded. 


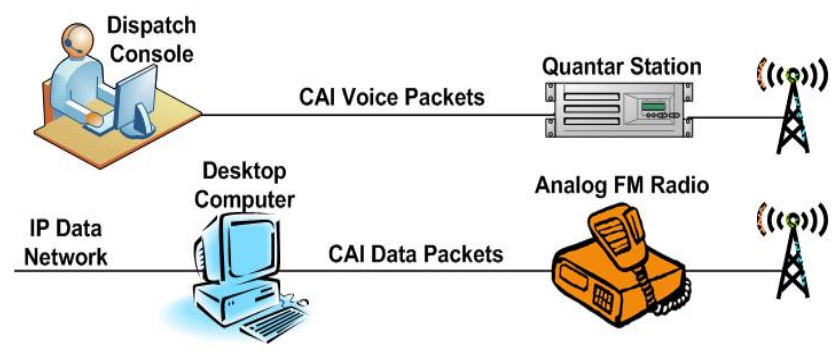

Figure 2 - Incorporated voice and data communication lines

Managing voice and data signaling on the same channel requires additional control logic. The control logic has two components: collision detection and packet repetition. Since the TDMA technique has been used and voice messages have priority over data packets, the base station listens to the radio channel and transmits data only when there is no voice signaling. However, a collision could happen when both a voice and a data messages are transmitted at approximately the same time. When a collision is detected, the collision detection component stops the transmission of the data message and grants priority to the voice message. The packet repetition mechanism retransmits the stopped data message and ensures its successful delivery once the channel is available for data transmissions.

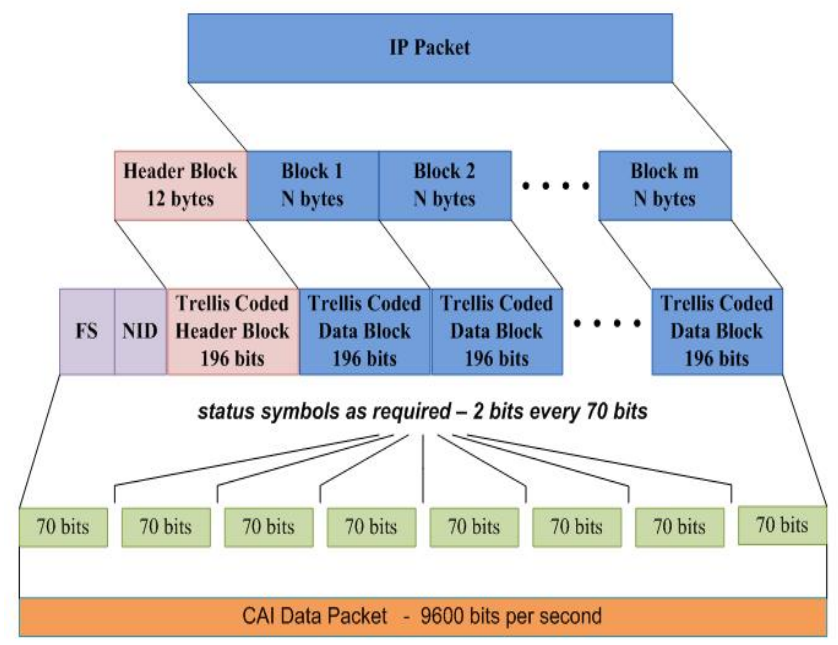

Figure 3 - CAI data packet to IP packet conversion

The decoding of IP data from CAI packets is illustrated in Figure 3. A CAI data packet is first broken into 70-bit segments separated by 2 status bits. These status bits are used to indicate the channel status and denote inbound and outbound messages. Each data packet starts with a frame synchronization word and a network identifier followed by a header and multiple data blocks. The frame synchronization sequence serves to identify the beginning of a new packet. The network identifier provides information about the network access code and the type of message (voice and data). Each header block is protected with a rate $1 / 2$ trellis code for error correction and contains the number of following data blocks. The following blocks are protected with rate $1 / 2$ or $3 / 4$ trellis codes, depending on whether the transmission is of the confirmed or unconfirmed type. Those decoded data blocks are then recombined resulting in the original IP data packet sent by the client. The maximum rate at which packets can be transmitted over the CAI is 9600 bits per second.

The transmission side of the base station implements the encoding of IP data into CAI packets. The transmitter developed by Ramsey [6] was combined with the newlydesigned receiver and control logic into a fully operational P25 data base station.

\section{TESTING}

Proper testing is an essential part of the completion of the project. We have divided the testing procedure into two parts: testing in laboratory conditions and testing in the field. The field tests will use an actual police vehicle radio setup, while in the laboratory we mimic the field setup using a digital radio and a laptop, called the "mobile client". For the laboratory testing, three types of tests have been designed: initial data tests with a mobile client, data tests with multiple mobile clients, and the assurance of voice priority. Currently, the base station is in the final testing stage which was designed to assure the priority of voice messages.

Each designed data test consists of 10,000 requests and tests were performed in both unconfirmed and confirmed type of operation. For each request, there were two packets (request and requested data) for unconfirmed and four packets for confirmed type (request, acknowledgement, requested data, and acknowledgement). The sizes of the packets varied between 12 bytes for the acknowledgement packet and 84 bytes for the requested data packet. The change in the rates at which the request packets were transmitted resulted in the change of the channel usage from $4 \%$ to $35 \%$. As a reference, the maximum channel usage observed was $22 \%$ when monitoring the activity on three separate $\mathrm{NH}$ police radio channels for a time period longer than a year. The channel usage during testing of $35 \%$ was high enough to simulate the utilization of the radio channel used by local NH police departments.

Initial end-to-end tests were essentially performed as blackbox tests, by passing IP request packets from a mobile client to the base station and transmitting requested data back to the mobile client [8]. In laboratory conditions, we expected to see the successful transmission of all data packets. However, during the tests some messages were lost. Tracing the packets using the white-box testing approach revealed improper alignment of the push-to-talk signal on the analog radio and packet synchronization preamble. In some situations the preamble was not long enough for the receiver to get in synchronization with the incoming packet. When these parameters were adjusted, hundreds of thousands of logged test messages demonstrated the proper transmission and reception of data packets at different rates. The laboratory testing setup is shown in Figure 4. 


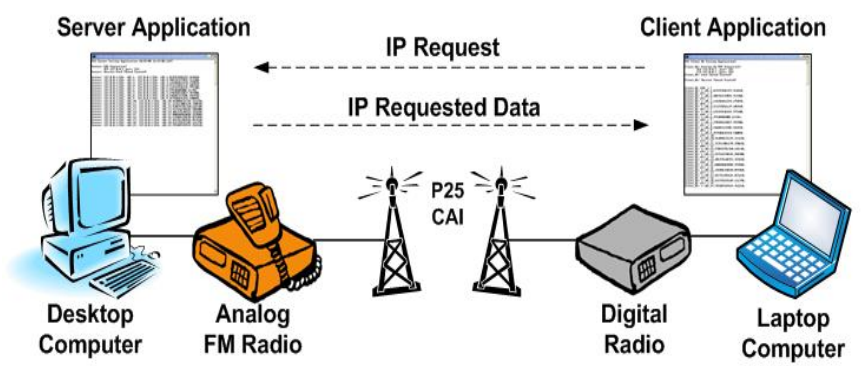

Figure 4 - End-to-end IP testing configuration

Once the tests with a single mobile client were successfully completed, the system was extended with additional mobile clients. In the tests with multiple clients, we expected to see collisions between data messages, since the clients communicate with the station simultaneously. The number of collisions was larger for the higher channel usage and varied between $0.015 \%$ for the channel usage of $4 \%$ and $1.61 \%$ for the channel usage of $35 \%$. These collided messages would be lost without packet retransmission. When a collision was detected or when the message was not received within a predefined time, the packet was retransmitted a maximum of four times at 15 second intervals. The 15 second intervals were later changed to a random time (10 to 20 seconds) to prevent consecutive collisions when multiple clients entered and left the waiting state at the same time.

Adding a new client to the system increases the radio channel usage which leads to a higher number of collisions. Handling collisions involves packet repetition and waiting time at the clients which slow down the overall communication speed. Presumably, a similar effect could be seen during combined voice and data transmissions in an emergency situation when the channel utilization reaches its maximum. The channel speed decreases when the communication is the most needed.

The multiple client tests showed a proper functionality of the collision detection component and with packet repetition all data messages were exchanged successfully between the station and multiple clients. However, prior to the field tests and real world deployment in a local police department with several cruisers, the system has to be shown to ensure the priority of voice communication and to satisfy QoS criteria. These final laboratory tests are currently underway.

\section{CONCLUSION AND FUTURE WORK}

Exchanging information in a digital way is very important for today's modern communication systems. Data messages would be beneficial for law enforcement departments to bring additional information into their cruisers. However, the commercially-available P25 data systems are not cost effective for small departments due to their limited budget. Also, first responders and mission-critical operations still cannot completely rely on the survivability and redundancy of publicly available services.
In this project we have designed a cost effective communication system which represents a good basis for small police departments to enable IP based data services to their vehicles. The system uses a personal computer and an analog radio for the reception and transmission of data packets. Data packets are transmitted together with voice messages over a single radio channel. This lowers the cost of providing data services by involving no additional wireless device in the vehicle, and increases security and reliability of data services since the radio infrastructure is owned and operated by the agency. Also, the system relies on the preexisting digital P25 compliant radio network which is standardized in the state of New Hampshire, and which provides a secure and reliable communication infrastructure for first responders.

Testing is a crucial part to the completion of the project. The laboratory testing configuration and overall testing strategy were presented. End-to-end tests showed promising results of successful transmission and reception of data packets among multiple clients in laboratory conditions. These results encourage us to continue with the testing procedures prior to the real world deployment.

The knowledge and data that we gathered during the testing stages should give a good insight to develop a complete model of a police radio channel. The model could be used to simulate and predict unwanted traffic behavior caused either by adding new clients to the system or by increasing the amount of traffic in an emergency situation.

The base station will be deployed in a local police department with several cruisers. It will be used for textual record queries initiated by the Project54 records application [9]. In combination with the Project54 speech user interface, this will provide a safer way to obtain vehicle and driver information on patrol [10] [11]. Since the data messages are of a shorter duration than voice messages, we believe that data queries will result in a reduction of the channel utilization as well. We will examine these effects in the future studies.

\section{ACKNOWLEDGMENT}

This work was supported in part by the U.S. Department of Justice under grants 2005CKWX0426 and 2006DDBXK099.

\section{REFERENCES}

[1] N. Jesuale, "Spectrum policy issues for state and local government," International Journal of Network Management, vol. 16, no. 2, pp. 89-101, 2006.

[2] D. Staehle, K. Leibnitz, and K. Tsipotis, "QoS of internet access with GPRS," Wireless Networks, vol. 9, no. 3, pp. 213-222, 2003. 
[3] Project 25 FDMA Common Air Interface New Technology Standards Project Digital Radio Technical Standards, Telecommunications Industry Association TIA\EIA-102.BAAA, 1998.

[4] Tyco Electronics. Expanding Digital Communication, $P 25^{I P}$ VIDA Network System. Massachusetts, USA : Tyco Electronics M/A-COM.

[5] Etherstack, "APCO P25 Base Station," Amsterdam, Etherstack, Inc.

[6] E. Ramsey, W. T. Miller, III, and A. L. Kun, "A Software Based Implementation of an APCO Project 25 Compliant Packet Data Transmitter," 2008 IEEE International Conference on Technologies for Homeland Security, Boston, MA, May 12-13, 2008.

[7] W. Stallings, Data and Computer Communication. Upper Saddle River, NJ: Parson Prentice Hall, 2007.

[8] J. Z. Gao, H.-S. J. Tsao, and Y. Wu, Testing and Quality Assurance for Component-Based Software. Norwood, MA: ARTECH HOUSE, INC, 2003.

[9] A. L. Kun, W. T. Miller, III, and W. H. Lenharth, "Computers in police cruisers," Pervasive Computing, IEEE, vol. 3, no. 4, pp. 34-41, 2004.

[10] Z. Medenica and A. L. Kun, "Comparing the influence of two user interfaces for mobile radios on driving performance," Driving Assessment 2007, Stevenson, WA, 2007.

[11] C. Maria and Z. Lorna, "Usability on patrol," in CHI '07 extended abstracts on Human factors in computing systems San Jose, CA, USA: ACM, 2007, pp. 1709-1714. 